\title{
Condiciones y experiencias de vida de las mujeres gestantes indigentes que viven en el casco noroeste metropolitano de San José ${ }^{1}$
}

Mónica Granados Hernández ${ }^{2}$ Johann Ramírez Campos ${ }^{3}$

Institución: Universidad de Costa Rica

\section{COMO CITAR}

Granados-Hernández, M. y Ramírez-Campos, J. (Octubre, 2013) Condiciones y experiencias de vida de las mujeres gestantes indigentes que viven en el casco noroeste metropolitano de San José. Rev. Actual de Costa Rica, 25, 1-16. Recuperado de: $<$ http://www.revenf.ucr.ac.cr/indigencia.pdf> ISSN 1409-4568

\section{RESUMEN}

El objetivo de este artículo es presentar los resultados de una investigación acerca de las condiciones y experiencias de vida de las mujeres indigentes. Participaron cuatro mujeres gestantes indigentes. La investigación se enmarcó en un diseño cualitativo de tipo fenomenológico. La recolección de los datos se ejecutó mediante la aplicación de la entrevista a profundidad, empleando la grabación para realizar observaciones repetidas; luego se analizaron los datos y se contrastó con el marco referencial. Entre las peculiaridades más relevantes que forman el perfil de una mujer embarazada indigente se encontró como factores comunes alcoholismo, drogadicción, prostitución, violencia física, sexual, psicológica y la delincuencia. Se concluye que las condiciones de vida están entrelazadas por las experiencias de vida de las mujeres embarazadas indigentes, condición a partir de la cual se determina su perfil.

Palabras clave: condiciones-de-vida, experiencias-de-vida, embarazo, indigencia, fenomenología

\footnotetext{
${ }^{1}$ Fecha de recepción: 15 de mayo 2013

Fecha de aceptación: 20 de julio 2013

2 Estudiante de la Escuela de Enfermería de la Universidad de Costa Rica. Correo electrónico: monic_gra07@hotmail.com
${ }^{3}$ Estudiante de la Escuela de Enfermería de la Universidad de Costa Rica. Correo electrónico: joh07cr@hotmail.com
} 


\title{
Conditions and life experiences of indigent pregnant women living in the northwest metropolitan of
} San José ${ }^{1}$

\author{
Mónica Granados Hernández ${ }^{2}$ \\ Johann Ramírez Campos ${ }^{3}$
}

Institution: Universidad de Costa Rica

\section{CITED}

\section{ABSTRACT}

The aim of this paper is to present the results of an investigation into the conditions and life experiences of indigent women. Involved four indigent pregnant women. The investigation was prompted by a phenomenological, qualitative design. The data collection was carried out by applying depth interviews, using recording for repeated observations, then the data were analyzed and contrasted with the framework. Among the most important characteristics that form a profile of a indigent pregnant woman found common factors: alcoholism, drug addiction, prostitution, physical violence, sexual and psychological and crime. We conclude that the conditions of life are intertwined with the life experiences of homeless pregnant women, from the condition which determines their profile.

Keywords: life-conditions, life-experiences, pregnancy, indigent women, phenomenology-research.

\footnotetext{
${ }^{1}$ Date of receipt: May 15, 2013

Date of acceptance: July 20, 2013

${ }^{2}$ Estudiante de la Escuela de Enfermería de la Universidad de Costa Rica. Correo electrónico: monic_gra07@hotmail.com

${ }^{3}$ Estudiante de la Escuela de Enfermería de la Universidad de Costa Rica. Correo electrónico: joh07cr@hotmail.com
} 


\section{INTRODUCCION}

En la actualidad, la indigencia es un tema de gran relevancia dentro de cualquier sistema social, el cual abarca muchos aspectos que quizá en distintas ocasiones son desconocidos; por ende, suele percibírsele solo como sinónimo de extrema pobreza y se deja de lado temas significativos para quienes viven este tipo de situación. Tal como menciona Barreat (2003), “...son muchos los sinónimos asociados a este término y entre ellos se pueden mencionar pobreza, penuria, privación, miseria, estrechez, dificultades, mendicidad, falta de recursos y/o medios para alimentarse, residenciarse, vestirse, entre otros.” (p.2).

Sin embargo, esa información desconocida incluye elementos que se relacionan de manera directa con estilos de vida saludable, abandono personal, abandono familiar y abandono social, por lo que muchas veces, esta condición de vida va más allá de la pobreza, dado que en ocasiones corresponde a una decisión tomada por el ser humano.

A pesar de que constituya una decisión personal, todo lo anterior genera una problemática en la que las condiciones de vida no favorecen a la población, produciendo, entre otras dificultades, el poco acceso a los servicios de salud, situación que se estudió en el presente trabajo, el cual se limita al grupo de mujeres gestantes del área metropolitana del país.

El objetivo de esta investigación fue analizar las condiciones y experiencias de vida que viven las mujeres gestantes indigentes del casco metropolitano en el año 2012.

\section{MATERIALES Y MÉTODOS}

Las participantes de ese estudio fueron cuatro mujeres adultas gestantes indigentes (sin importar su edad gestacional), del casco noroeste metropolitano de Costa Rica. Se llevó a cabo una selección por conveniencia del investigador debido a la dificultad de contactar con estas mujeres por su movilidad geográfica.

La investigación es cualitativa de tipo fenomenológico, dado que según Rojas (2008) “...el método fenomenológico estudia las realidades cuya esencia depende del modo en que es vivida y percibida por el sujeto, una realidad interna y personal, única y propia de cada ser humano." (p. 46).

Para enmarcar la investigación se aplicó el diseño metodológico propuesto por Joseph A. Maxwell, el cual se basa en cinco aspectos:

\section{Preguntas de investigación:}

¿Cómo aborda el sistema de salud a las personas en condiciones de indigencia?; ¿Qué tipo de necesidades tienen las mujeres indigentes?; ¿Qué tipo de necesidades tienen las mujeres embarazadas indigentes?; ¿Cómo son las condiciones de vida de las mujeres embarazadas indigentes?; ¿Cómo se establece la condición de indigencia en las personas adultas?; ¿Cómo se diferencia la indigencia de las condiciones urbano-marginal y extrema pobreza?; ¿Cómo se podría mejorar el manejo del embarazo en condiciones de indigencia?; ¿Dónde se podría 
acceder a las mujeres adultas indigentes?; ¿Qué tipo de instrumento se puede emplear para recolectar información en mujeres adultas indigentes?; ¿Qué tipo de estrategias se deben aplicar o tomar en cuenta a la hora de elaborar un instrumento de recolección de datos en las mujeres adultas indigentes?

\section{Propósito}

En esta investigación y con base en las preguntas anteriores se analizan las condiciones y experiencias de vida de las mujeres adultas indigentes en estado de gestación, con el fin de comprender el accionar del sistema de salud para este tipo de población, así como los cuidados que estas mismas mujeres tienen durante su periodo de embarazo.

\section{Contexto conceptual}

Se plantearon dos grandes unidades de análisis: la primera se refiere a las condiciones de vida y la otra, a las experiencias de vida de las mujeres adultas gestantes indigentes en el área metropolitana, razón por la que se seleccionó literatura (periódicos, libros, y artículos científicos) relacionada con la temática en estudio.

\section{Método}

Por las características del estudio, se aplicó el método fenomenológico, el cual está compuesto por cuatro etapas:

\subsection{Etapa previa}

Clarificación de presupuestos: se trabajó con población indigente, debido a que es una población de difícil acceso, una vez identificada se procede aplicar el consentimiento informado y luego, la entrevista a profundidad.

\subsection{Etapa descriptiva}

Elección de la técnica o procedimiento apropiado. Como lo establece el método fenomenológico, la entrevista a profundidad es la técnica que proporciona mayor cantidad de información. Esta entrevista fue grabada por los investigadores con el fin de realizar observaciones repetidas. Además, se observó la complejidad de todas las partes, excluyendo la observación selectiva.

Elaboración de la descripción protocolar. En esta parte se analizó el fenómeno, en este caso de la indigencia, en su contexto natural; es decir, en "plena calle", momento en el que los investigadores observan el proceso de "callejización" que produce la indigencia, por lo cual se observaron y analizaron conductas, actitudes, lenguaje corporal durante la entrevista.

\subsection{Etapa estructural}

Lectura general de la descripción de cada protocolo. En primera instancia se procedió a realizar una lectura general de cada protocolo y filtrar la información relevante en las entrevistas realizadas. Se delimitaron las unidades temáticas y se valoró el surgimiento de unidades emergentes. Luego se determinó el tema central que domina cada unidad temática y se elimina los datos no pertinentes para el estudio. Luego, se procedió a expresar 
lo acontecido en un lenguaje científico y, por último, se integraron todos los temas centrales en una estructura descriptiva particular que formó parte de una estructura general.

\subsection{Etapa discusión de los resultados}

Como parte de los antecedentes encontrados, se analizaron las conclusiones establecidas por otros investigadores respecto del tema social de la indigencia para contradecirlas, compararlas, entender mejor las posibles diferencias y enriquecer el área estudiada.

\section{Validez}

Para la validez de esta investigación se tomó en cuenta la intersubjetividad, puesto que es un aspecto valioso en una investigación cualitativa. A partir de dicho aspecto, los investigadores analizaron -de forma individual y conjunta- los datos obtenidos por medio de la aplicación estricta de los instrumentos para la recolección de datos.

Respecto de las unidades y categorías de análisis se estableció lo siguiente:

Unidad de análisis: las experiencias de vida de las mujeres gestantes indigentes.

Categorías: aspectos sociodemográficos.

Subcategorías: la edad, localización y procedencia, estado civil, hijos(as) y ocupación.

Unidad de análisis: las condiciones de vida de las mujeres gestantes indigentes.

Categorías: el acceso a los servicios públicos sanitarios y las condiciones de habitalidad.

Subcategorías: el control prenatal, tratamiento obstétrico y el estado físico.

Entre los instrumentos empleados para la recolección de datos se aplicó la técnica de entrevista a profundidad, la cual consiste en una conversación directa, abierta e informal entre el investigador y los consumidores para explorar e identificar los sentimientos más íntimos respecto del producto o las marcas en estudio.

Es directa porque se realiza de manera personal, cara a cara, entre el investigador y el investigado. Se analizan los resultados de las respuestas oralmente formuladas y el lenguaje no verbal de los entrevistados, tal como los gestos o las actitudes.

La entrevista en profundidad es abierta, porque emplea una guía de pautas para que el investigador conduzca la entrevista y formule preguntas espontáneas, no necesariamente planificadas, que surgen en ese momento y que son de utilidad para la investigación. Cada entrevista fue grabada con el fin de facilitar la comunicación, la recolección y evitar que haya sesgos; el tiempo para realizarla fue indefinido, con el objetivo de no intimidar a la persona entrevistada y para fomentar la empatía entre ambos (investigado/investigador, de modo que se sustraiga la mayor cantidad de información. 


\section{Revista Electrónica Enfermeria Actual en costa Rica}

La guía de entrevista se constó de preguntas generadoras, de cuyos temas fueron definidos por las categorías de las distintas unidades de análisis, las cuales se utilizaron para analizar los resultados.

\section{Consideraciones éticas}

Se aplicó el consentimiento informado, en el que se les indicó a las personas participantes en la entrevista acerca de los fines de la investigación; también se les informó que la información es totalmente confidencial, que solo se empleará para fines educativos y que solo los investigadores accederán a los datos. Después del procesamiento de la información, esta será publicada y se mantendrá el anonimato.

Por otra parte, los investigadores consideran las necesidades de cada uno de los sujetos de estudio, a quienes se les garantizó un ambiente adecuado para el desarrollo de la entrevista, de modo que respondan a las preguntas y se obtengan los resultados más precisos que cumplan con los objetivos de la investigación.

\section{RESULTADOS}

\section{Experiencias de vida de las mujeres gestantes indigentes.}

Entre los aspectos sociodemográficos se consideró la edad, el lugar de procedencia, el estado civil, el número de hijos y la ocupación, características independientes de la indigencia, lo que se corrobora al observar en conjunto las características sociodemográficas de las mujeres entrevistadas. En cuanto a la residencia, las cuatro personas entrevistadas no tienen un sitio específico para dormir; no obstante, las entrevistadas dos y tres asisten a una de las organizaciones nacionales conocida como Centro dormitorio y de atención primaria para habitantes de calle, ubicada entre las avenidas 3 y 5 de la ciudad de San José.

Las otras dos mujeres no asisten a ningún centro de ayuda, sino que se ubican en distintas regiones del casco noroeste metropolitano de San José, lo cual se afirma cuando una de ellas mencionó lo siguiente: “...bueno, aquí los hombres les gusta llevarme a tomar algo y bueno, ya después a veces como me ofrecen un poco de dinero me voy con ellos." (Entrevistada 4).

Respecto de la ocupación obsérvese la siguiente información:

\begin{tabular}{cl}
\hline Entrevistada & \multicolumn{1}{c}{ Ocupación } \\
\hline 1 & $\begin{array}{l}\text { Distribución de drogas y } \\
\text { actividad sexual remunerada }\end{array}$ \\
\hline 2 & $\begin{array}{l}\text { Colaboración en el centro } \\
\text { dormitorio }\end{array}$ \\
\hline 3 & $\begin{array}{l}\text { Venta de comidas en } \\
\text { construcciones }\end{array}$ \\
\hline
\end{tabular}


Otro aspecto que se investigó es la cantidad de hijos, obsérvese la siguiente información:

\begin{tabular}{ccl} 
Entrevistada & $\begin{array}{c}\text { Edad cuando tuvo el } \\
\text { primer hijo }\end{array}$ & \multicolumn{1}{c}{ Número de hijos } \\
\hline 1 & 14 años & $\begin{array}{l}\text { Tiene un hijo de 18 años de edad; } \\
\text { en la actualidad, está embarazada. }\end{array}$ \\
\hline 2 & 17 años & $\begin{array}{l}\text { Tiene dos hijos; en la actualidad, } \\
\text { está embarazada. }\end{array}$ \\
\hline 3 & 13 años & $\begin{array}{l}\text { Tiene tres hijos; en la actualidad, } \\
\text { está embarazada. }\end{array}$ \\
\hline 4 & 26 años & Está embarazada. \\
\hline
\end{tabular}

En este aspecto se destaca que el haber sido jóvenes madres por distintas causas dificultó aún más sus circunstancias; por ejemplo, no cuentan con el soporte de sus familias u otras redes de apoyo, condición que las obliga a vivir en las calles.

El estado civil también se investigó, dado que la indigencia afecta tanto a mujeres solteras, como casadas, viudas, divorciadas y en unión libre. Cabe destacar que la inestabilidad sentimental y familiar es un elemento común entre las cuatro mujeres entrevistadas: la entrevistada tres comentó que ha estado casada dos veces, la primera vez enviudó y la segunda vez se divorció para comenzar una relación en unión libre con su actual pareja, padre del bebé que está gestando, mientras que las otras participantes (uno y cuatro) son solteras.

A partir del lugar de procedencia, el estado civil, la cantidad de hijos, entre otros, se comprende mejor el origen de muchas experiencias que determinan las condiciones de vida del ser humano, razón por la que se indaga acerca de los aspectos sociodemográficos de las cuatro mujeres participantes para abarcar la siguiente categoría, basada en las experiencias de vida.

Las experiencias de vida en todo ser humano influyen -de cierta manera- en su condición de vida posterior; por ende, es indispensable analizarlas para comprender la condición en que viven las personas entrevistadas. Como parte de los factores desencadenantes de la indigencia se encuentran las diversas adicciones a sustancias psicoactivas, como alcohol, tabaco, crack (piedra) y cocaína; así como un inadecuado manejo de los procesos emocionales, conflictos familiares, rechazo social y discriminación dentro del grupo de pares. Lo anterior es corroborado por Santillana, psicóloga, cuando expresa que,

una persona puede caer en la indigencia por diversas razones entre las que destacan el padecimiento de trastornos mentales como la esquizofrenia. Sin embargo, asegura que cada vez es más común observar que las depresiones severas sin tratamiento a tiempo y adicciones por el alcohol y las drogas están empezando a ubicarse en los primeros lugares de la lista de factores que influyen para que un ser humano caiga en la indigencia (Umbría; 2011, p.1).

La afirmación anterior, lo confirma la entrevistada dos que dice: “Casi la mayoría de la gente que consume o tiene problemas con la familia los dejan botados; siempre es por eso, casi la mayoría es por eso. Entonces uno dice, ijuepucha! con todo lo que le pasa a uno, que nadie lo quiere a uno y como uno consume como que todo el 


\section{Revista Electrónica Enfermería Actual en costa Rica}

mundo lo rechaza y entonces a uno le da lo mismo: que si se baña que si no se baña, que se moja o no se moja, que si anda sucio, ya se hace costumbre. Entonces va más o menos por ahí. Y si usted les hace una entrevista a todas las personas que consumen, va por ahí.” (Entrevistada 2).

Otro aspecto que es parte de la problemática social se refiere al abuso sexual, tomando en cuenta que “...internacionalmente se reconoce el abuso sexual y la explotación sexual comercial como problemas de salud pública, como violación de los derechos humanos y obstáculo para el desarrollo" (CICAES, 2006; p.6).

Las experiencias de vida de estas mujeres embarazadas indigentes brindan un mejor panorama de las situaciones que determinan su entorno, en las que se incluye las condiciones que les generan situaciones adversas para el desarrollo óptimo de sus vidas y las de sus hijos e hijas. En la siguiente unidad de análisis se presentan las condiciones de vida que estas mujeres tienen durante su etapa de gravidez.

\section{Condiciones de vida de las mujeres gestantes indigentes.}

Esta categoría se refiere a los servicios de atención en salud que se brindan en Costa Rica, respecto de lo que es necesario destacar que un Sistema Nacional de Salud debe incluir la totalidad de los componentes del sistema social, los cuales se ligan, en forma directa o indirecta, con el bienestar de la población. En relación con lo anterior, se determina que el sistema de salud de Costa Rica cubre a todas las personas que acuden a un servicio sanitario, sin importar su condición, particularidad a la que se refiere la persona número dos, quien asiste a una clínica para llevar un control prenatal: "Mi bebé no se ha dejado ver, bueno para ver si es chiquito o chiquita (...). Al principio fui como a dos citas, pero no volví porque ya perdí una cita y en la clínica como que cuesta que le den una cita. Y tengo como tres meses pidiendo que me atiendan y hasta hoy logré que me volvieran a atender." (Entrevistada 2).

Como parte de las barreras que impiden a las gestantes acceder a un servicio de salud cabe destacar el déficit de conocimiento de las mujeres indigentes así como su bajo nivel económico; tal es el caso de la entrevistada cuatro, quien comenta: “...yo no llevo ningún control, aunque sí quiero ir para que todo vaya bien, pero como estoy empezando y no tengo mucha plata no he ido..." (Entrevistada 4).

Lo anterior es relevante en el sentido de que el estado físico de las mujeres entrevistadas puede aumentar o disminuir las posibilidades de presentar un embarazo de alto riesgo, razón por la que, según Chávez (2006),

"existe mayor probabilidad de mortalidad y morbilidad, tanto para el recién nacido (a) como para la madre; por consiguiente, se destaca la trascendencia de un modelo de atención y cobertura para las mujeres indigentes, dado que podrían mejorar su condición de vida, máxime considerando que el "modelo readecuado de atención es un componente clave de la reforma sectorial y su impacto se puede valorar objetivamente a través de varias dimensiones, entre las que destacan las siguientes: acceso y cobertura, integralidad, calidad y equidad en la atención." (Ministerio de Salud de Costa Rica et al., 2004, p.34). 
En cuanto a las condiciones habitacionales hay problemáticas relacionadas con la inseguridad, el rechazo social o discriminación y las dificultades para satisfacer las necesidades básicas del ser humano, lo cual se confirma en las palabras de la entrevistada tres (2012): "Es que me asaltaron, me robaron todo (...) en las palmeras, en el parque La Merced (...) es que ahí es muy peligroso, es muy peligroso caminar."

Por otra parte, existen organizaciones que brindan servicios con el fin de mejorar las condiciones de vida de las personas indigentes; sin embargo, no representa una solución absoluta a las problemáticas mencionadas, dado que la adicción a sustancias psicoactivas por parte de estas personas genera un desinterés en relación con las condiciones habitacionales. En ocasiones, dichos centros no cuentan con los recursos suficientes para cubrir la demanda de albergue o refugio.

La entrevistada dos (2012) comenta que desde hace tres años conoce el centro dormitorio, lugar que funge como refugio y en el que ha hallado oportunidades de estudio y trabajo; sin embargo, ella mencionó que desde su niñez convive con condiciones de habitalidad poco favorables que han desembocado en problemas de adicción a las drogas, violencia física e inseguridad, experiencia que manifiesta a continuación: "Yo vivía con mi mamá y mis hermanos, pero ella trabajaba día y noche, día y noche, por eso estábamos en la calle a pesar de tener casa (...) cuando no teníamos nada le pedíamos a los vecinos (...) y yo consumía cuando estaba en la calle (...). Y digamos, usted sabe que cuando uno está en la calle y uno es mujer siempre sale quien lo invite. O si no, uno en la calle es otra cosa porque uno anda asaltando a todo el mundo y todo, entonces no se le hace complicado porque, diay, a uno le da lo mismo todo. Pero ya tengo cuatro años de no consumir nada (Entrevistada 2).

La condición mencionada se relaciona, a su vez, con la de pobreza y mendicidad, “...partiendo del concepto de pobreza como un síndrome situacional en que se asocian el infraconsumo, la desnutrición, las precarias condicionales de vivienda, los bajos niveles educacionales, las malas condiciones sanitarias." (Larraín, F., 2008, p.104). La mendicidad es un fenómeno social en el que la persona busca ayuda económica pública; en el ámbito educativo las personas con escasos recursos, extrema pobreza, precariedad, indigencia, entre otras, sufren de exclusión social por los mismos sistemas educativos, así como por los grupos de pares, situación que los motiva a abandonar sus estudios. En este aspecto expresó la entrevistada dos que antes, cuando visitaba el centro dormitorio, estudiaba en un instituto, en el cual se encontraba cursando sétimo y octavo de secundaria y un curso de computación; sin embargo, tuvo que abandonarlos por la dificultad para pagarlos que, a su vez, le generaba problemas en el lugar y limitaba su aprendizaje.

Por otra parte, el escenario que rodea a una persona indigente muchas veces está relacionado con la drogadicción -tal como se ha mencionado -. El consumo frecuente promueve que las personas generen tolerancia, por lo que la búsqueda y adquisición de drogas disminuye las oportunidades para dichos individuos, entre las que se incluyen el acceso a un adecuado nivel educativo y económico. En el caso de la entrevistada tres, ella es bachiller en Contabilidad; sin embargo, perdió su título por consumir drogas, tal como se refleja en la siguiente cita: "Me gradué en el Enmanuel Mongalo y Rubio, en Nicaragua, pero es que perdí el título, toqué fondo, lo perdí por drogas. ¡Es que yo no pagué, ¿entiende?! ¡Si no pago me quitan el título!” (Entrevistada 3).

El ser humano demanda necesidades basadas en aspectos individuales y colectivos; como consecuencia, es fundamental analizar temas como la alimentación y nutrición, vestimenta, esparcimiento, seguridad, relaciones interpersonales y relaciones familiares. Las cuatro entrevistadas viven en condiciones de pobreza, notables en la 


\section{Revista Electrónica Enfermería Actual en costa Rica}

dificultad ocasional para satisfacer necesidades básicas como la alimentación, característica de la pobreza que se refiere al infraconsumo, concepto que sostiene que pobre no es quien tiene poco, sino aquel que no tiene lo suficiente para satisfacer las necesidades básicas que experimenta en un determinado momento, a saber deficiencia en la satisfacción de alimentos, agua potable, sanidad, vestimenta, entre otros aspectos.

Lo anterior se manifiesta en las palabras de la entrevistada cuatro cuando dijo: "Bueno, comía de lo que me encontrara y así, a veces iba a pedir o nada más me aguantaba el hambre, y como ya le dije a veces iba a un centro de por ahí, entonces me bañaba o a veces ni me importaba y pasaba muchos días sin bañarme." (Entrevistada 4).

Es fundamental rescatar que la alimentación durante el embarazo es indispensable para el desarrollo materno fetal; sin embargo, como se mencionó, la extrema pobreza, ligada a la condición de indigencia de estas personas, demuestran que hay alimentación inadecuada y desconocimiento acerca del desarrollo del embarazo, aunque los conflictos familiares también afectan a las gestantes, debido a la vulnerabilidad nutricional y necesidades que el proceso de embarazo genera.

Tal como se mencionó, los conflictos familiares representan uno de los motivos que desembocan la indigencia: “...las personas en indigencia refieren haber llegado a esta condición por la interrelación entre conflictos o pérdidas familiares, un inadecuado manejo de sus procesos emocionales y la farmacodependencia y/ o el alcoholismo." (Rojas, 2006; pp.5-6). Dicha problemática se refleja en la experiencia de vida de la entrevistada número tres, quien sufrió maltrato familiar por parte de sus hermanos, lo cual se evidencia en la siguiente cita: "Mis hermanos me marginaban, porque yo siempre tomaba (...) yo siempre tomaba en la 15 de setiembre ahí por el Rancho de Guanacaste, hasta que mi hermano me echó de la casa, y es que yo soy hija única, ellos todos son varones (Entrevistada 3).

En algunos casos, los conflictos familiares, provocan que la persona se mantenga en la condición de indigencia, estableciendo relaciones sentimentales difíciles y crisis emocionales, debido a que no cuentan con una red de apoyo sólida ni con experiencias para desarrollar habilidades en el manejo de conflictos con otros individuos, ni para establecer soluciones efectivas en situaciones de estrés, una particularidad que contribuye a que la persona indigente se convierta en víctima de delincuencia, robos y agresión física y mental; como en el caso de la entrevistada tres, quien comentó: "Es que me asaltaron, me robaron todo (...) en las palmeras, en el parque La Merced (...) es que ahí es muy peligroso, es muy peligroso caminar.” (Entrevistada 3).

En el caso del empleo, se define con base en las actividades que representen la obtención de un lucro o alguna remuneración económica por parte de las mujeres entrevistadas. La entrevistada uno comenta que ella se dedica a vender drogas y a la actividad sexual remunerada; la entrevistada dos se encargaba de ayudar en la limpieza del centro dormitorio durante su estadía; sin embargo, en la actualidad no participa en actividades laborales. La entrevistada 3 expresó que ella se encargaba de la limpieza de un casino en San José centro y la entrevistada cuatro mencionó dedicarse a actividades sexuales remuneradas, en las cercanías del mercado "El Paso de la Vaca" con el objetivo de mejorar su condición actual como habitante de calle. 


\section{Revista Electrónica Enfermería Actual en costa Rica}

\section{DISCUSIÓN}

Como trabajadores de la salud integral, el y la profesional en enfermería no debe ser indiferente respecto de su cotidianeidad; en el caso de la indigencia, según Chacón (2008),

“...es una manifestación social que enfrentan día tras día gran número de personas sin distinción de edad, género o estatus social en el mundo. Costa Rica no es la excepción, dado que cada vez es más frecuente encontrar personas que convierten las calles en su hogar, desarrollando su cotidianeidad en las condiciones más adversas, constituyéndose en una condición que representa una de las mayores violaciones a los derechos humanos. (s.p.)

En relación con lo anterior cabe destacar que tal como explica la psicóloga Berta Santillana, quien lleva más de 30 años ejerciendo su profesión en el estado Monagas, mencionado por Umbría (2011) una persona puede caer en la indigencia por diferentes razones, entre las que destacan el padecimiento de trastornos mentales como la esquizofrenia. Sin embargo, asegura que cada vez es más común observar que las depresiones severas sin tratamiento a tiempo y adicciones por el alcohol y las drogas están empezando a ubicarse en los primeros lugares de la lista de factores que influyen para que un ser humano caiga en la indigencia.

Además, lo anterior se relaciona con que “...en la década de 1980, la Organización de las Naciones Unidas (ONU) estimó que había una población de más de cuarenta millones de niños y niñas de la calle en América Latina." (Shaw, 2011, p. 24).

Según lo expuesto, las calles de Costa Rica cada vez más se convierten en el albergue de muchas personas, lo cual significa que muchos de nuestros habitantes se están enfrentando al frío, la lluvia y otras condiciones, sin un techo en el cual albergarse, pensando también la realización de las necesidades básicas del ser humano, dígase, alimentación, evacuación intestinal y urinaria, higiene personal.

Por otro lado, dada la imagen que se ha construido acerca del indigente como personas que andan mal vestidas o harapientas, huelen mal, piden en las calles, permanecen en las aceras acostados; al respecto, es necesario incluir la definición aportada por Rojas (2006) que las personas en indigencia se caracterizan por los espacios donde desarrollan su vida cotidiana y por las actividades que realizan presentan características como: haber llegado a esta condición por la interrelación entre conflictos o pérdidas familiares, un inadecuado manejo de sus procesos emocionales y la farmacodependencia y/ o el alcoholismo, a lo que se agrega que tales adicciones, en su mayoría, aparecen junto a la indigencia y; por ende, muchas veces encaminan a la persona en la delincuencia o, como es el caso de algunas mujeres indigentes, a la prostitución, actividad para obtener dinero en su entorno.

Tienden adormir en aceras, lotes baldíos o al lado de los comercios del centro de San José y deambulan para suplir sus necesidades por la zona noroeste del casco metropolitano y algunos barrios del sur del cantón de San José.

Estas personas tienen apariencias muy diversas, dado que algunos pueden estar cerca de la imagen clásica de un mendigo, cuyas características principales son la falta de aseo y en algunos casos de zapatos, la ropa sobrepuesta, la barba y el pelo largo; otras pueden confundirse con cualquier transeúnte, dado que van a 
organizaciones no gubernamentales de asistencia en donde diariamente, se rasuran, cambian de ropa y se cortan el pelo y las uñas, además de que cargan algunas pertenencias, lo cual se refleja en la siguiente cita:

La mayor parte de estas personas sufre de diversas adicciones, principalmente al alcohol, tabaco $\mathrm{y}$ crack. Estas personas se bañan, consiguen alimentos y ropa en organizaciones no gubernamentales que ofrecen este tipo de servicios, aunque también dicen comprar alimento y ropa" (Artavia y Astúa, 2000, p. 164).

Las actividades productivas de estas personas son variadas, entre ellas se encuentran: la mendicidad, el trabajo sexual, el reciclaje de papel, latas vacías y desechos de cobre o aluminio, traslado de carga de los comercios, limpieza de zapatos, lavado y cuidado de carros o de comercios en las noches, venta de drogas y robo. Tal como se colige, muchas veces estas personas pueden confundirse o pasar desapercibidas, debido a las fuentes de ayuda a las que recurren para cubrir sus necesidades básicas por un tiempo; sin embargo, también existen factores de riesgo que incitan a las personas a caer en este estado de callejización, tales como prostitución, delincuencia, drogadicción, alcoholismo, etc.

\section{Según Vargas (2008),}

“...en las calles de la capital de Costa Rica y su periferia viven 2000 personas prisioneras del crack, afectadas por un severo grado de adicción a esa droga. De ese total, de acuerdo con el ministro de Seguridad Pública, Fernando Berrocal, 600 tienen antecedentes delictivos y sacian su necesidad de drogas con el dinero que obtienen de asaltos y robos." (s.p.)

De igual forma, la indigencia no solo representa una problemática para quienes la viven de manera directa, sino para los costarricenses en general, dado que, de acuerdo con Loaiza (2007), al menos siete puntos de San José están tomados por grupos que ahuyentan a peatones y conductores. En la minúscula capital hay zonas vedadas para los turistas. Acercarse a calle 12 o a los linderos del viejo cine Líbano, en la calle 10, es ofrecerles a los ladrones la cartera, la cámara o el celular.

Entonces, se habla de que en nuestro territorio es peligroso transitar y la paz ha sido perturbada; no obstante, analizando el panorama en general, cabe preguntarse: ¿es acaso culpable ese hombre o esa mujer que camina por las calles buscando asaltarme para obtener dinero?, ¿es acaso el gobierno culpable de esta situación? O por el contrario, ¿somos acaso nosotros los culpables por nuestra indiferencia? Esta clase de reflexión viene a nuestra mente en cada momento; por tanto, hemos decidido indagar aún más, establecer cuáles son las condiciones, las experiencias de vida que tienen estas personas en estado de indigencia. Otra pregunta que se formuló es la siguiente: ¿Es la indigencia machista como nuestra sociedad? Más bien, ¿existirá diferencia alguna entre hombres y mujeres indigentes? Y aún más, ¿qué pasa en el caso de algunas mujeres indigentes?

Como se mencionó, uno de los problemas que genera la indigencia es la prostitución: si pensamos en hombres satisfaciendo sexualmente a una mujer por dinero, el riesgo que puede presentar es alguna infección de transmisión sexual, al igual si el caso fuera de un hombre que satisface a otro hombre, pero ¿qué sucede en el caso de una mujer indigente?, ¿qué pasa si queda embarazada? La idea de una mujer indigente y embarazada es 
sumamente preocupante: como enfermeros y enfermeras todo el proceso de gestación nos es relevante, razón por la que pensamos en todo lo que implica el estado de gravidez en una mujer que vive esta situación social.

Retomando lo anterior, la prostitución es el deseo de saciar el hambre sexual en una persona que voluntariamente muestra en venta su cuerpo a cambio de dinero; no obstante, muchas de las mujeres que encontramos en las calles son vulnerables y en algunos casos son obligadas a desempeñarse en dichas actividades comerciales; por ende, es correcto afirmar que existe un grado de violencia, ya sea, física, sexual o psicológica para obligarlas a que accedan.

Grandi, Luchtemberg, y Sola (2007) afirman que “...en estudios recientes se ha señalado que el patrón de la ganancia de peso durante el embarazo, especialmente entre el segundo y tercer trimestre se asocia al crecimiento fetal, independientemente del estado nutricional materno pregestacional”. (p.p. 677-684).

Ahora bien, esa ganancia de peso, a pesar de ser un cambio normal de la condición en que se encuentra la mujer, no puede dejarse de lado, debido a que los hábitos nutricionales son esenciales en el proceso, lo cual se podría apoyar en lo mencionado por Uauy, Atalah, Barrera y Behnke (2002), quienes definen algunos riesgos que se pueden encontrar si estos hábitos no son los adecuados:

El embarazo constituye una de las etapas de mayor vulnerabilidad nutricional en la vida de la mujer. Existe una importante actividad anabólica que determina un aumento de las necesidades nutricionales en relación con el periodo preconcepcional. La desnutrición materna pregestacional o durante el embarazo se asocia con un mayor riesgo de morbimortalidad infantil, en tanto que la obesidad también constituye un factor importante de riesgo, al aumentar algunas patologías del embarazo, la proporción de niños macrosómicos y por ese mecanismo, las distosias y complicaciones del parto (p.1).

Un adecuado aporte nutricional es imprescindible, puesto que si la madre del futuro bebé no se alimenta bien, este es propenso a presentar factores de riesgo durante el proceso de gestación, relacionados con el crecimiento fetal. Tal como afirman Gómez y Manuel (sf), el control del embarazo en Atención Primaria pretende la preparación global para la maternidad, influyendo en una etapa de especial vulnerabilidad para la salud materno-fetal en la que, además, existe una mayor receptividad materna para las actividades de educación para la salud.

Al igual que el aporte nutricional, el control del embarazo es más que relevante para desarrollar un óptimo proceso gestacional, para ello se debe brindar educación a la madre y al padre relacionada con el cuido de su futuro bebé; mas en el caso de personas indigentes, es difícil que acudan a un centro hospitalario para atención prenatal, lo cual se corrobora en la siguiente cita:

El control prenatal (CPN) es un conjunto de acciones que involucra visitas de parte de la embarazada a la institución de salud y la respectiva consulta médica, con el objetivo de vigilar la evolución del embarazo, detectar tempranamente riesgos, prevenir complicaciones y prepararla para el parto, la maternidad y la crianza. La Organización Mundial de la Salud (OMS) considera que el cuidado materno es una prioridad que forma parte de las políticas públicas como 


\section{Revista Electrónica Enfermería Actual en costa Rica}

estrategia para optimizar los resultados del embarazo y prevenir la mortalidad materna y perinatal. Sin embargo, no todas las mujeres acceden al CPN y algunas no logran adherirse a las recomendaciones, ni cumplir con la periodicidad de asistencia. Según la hipótesis, existen barreras ajenas a las gestantes que les impiden acceder y adherirse al CPN (Cáceres, 2009; p. 1).

A partir de lo expuesto, es evidente que la mujer indigente y embarazada se enfrenta a muchos riesgos, tanto para ella como para el bebé, lo cual lo confirman Delgado y López (2004):

Está claro por abundantes estudios publicados que el tabaco se relaciona con determinadas complicaciones obstétricas, como aborto, embarazo ectópico, abruptio placentae, placenta previa, parto pretérmino y CIR. En este sentido, los hijos de madres fumadoras (con 20 o más cigarros/día) presentan entre 200-300 g menos que los de las no fumadoras. Los hijos de madres alcohólicas pueden presentar el síndrome de alcohol fetal (SAF), caracterizado por CIR, dismorfia facial, retraso mental y alteraciones diversas (659-669).

Asimismo,

El consumo de drogas y medicamentos puede ser perjudicial para el desarrollo del embarazo, y una mujer que abusa de ambos puede experimentar un mayor número de complicaciones, debido a su estilo de vida. Las mujeres que abusan de algunas sustancias en general manifiestan déficit nutricional; también puede observarse anemia y retraso del crecimiento fetal. La mujer embarazada que abusa de drogas o medicamentos corre un mayor riesgo de preeclampsia (Curtis y Schuler, 2003, p.252).

De la información se colige que de las condiciones en que una mujer puede llevar su embarazo, la indigencia no es la mejor, máxime que los porcentajes demuestran que son pocos los casos en los que el embarazo concluye de manera satisfactoria o en los que el bebé nace sano; por ende, como profesionales, sus condiciones sociales así como la situación prenatal son aspectos que competen a los trabajadores de la salud -en este caso particular a enfermería- para que basados en investigaciones sean agentes que propongan estrategias de apoyo y trabajo a los generadores de políticas sociales y de salud que disminuyan la complejidad de la indigencia sumado a mujeres gestantes.

\section{CONCLUSIONES}

Las condiciones de vida en las que una mujer indigente embarazada se desarrolla depende de diversos factores, personales, sociales, económicos y emocionales. A pesar de que la indigencia va de la mano de la pobreza, muchas de estas mujeres embarazadas cuentan con ciertos recursos económicos para solventar su situación. Sin embargo no es suficiente el apoyo y esfuerzo que hacen algunas instituciones que dan auspicio a personas en condición de indigencia, las cuales dan preferencia a las mujeres embarazadas indigentes.

Las condiciones diarias que las mujeres gestantes indigentes enfrentan están determinadas por sus experiencias de vida, dentro de las cuales destacan: alcoholismo, drogadicción, prostitución, violencia física, sexual, psicológica y delincuencia, denominadores comunes de la indigencia. Todas las características mencionadas influyen en gran medida para la caracterización del perfil de estas mujeres. 


\section{Revista Electrónica Enfermería Actual en costa Rica}

\section{REFERENCIAS}

Artavia X., Astúa, I. (2000). Estudio exploratorio sobre las características de las personas en condición de indigencia localizadas en el casco noroeste metropolitano y algunos barrios del sur del cantón central de San José. Ejército de Salvación, San José.

Barreat, Y. (2003). Un síndrome biosicosocial. Recuperado de http://www.saber.ula.ve/bitstream/123456789/14897/1/capitulo16.pdf

Cáceres, F. (2009). Control prenatal: una reflexión urgente. Revista Colombiana de Obstetricia y Ginecología. 60 (2), 1. Recuperado de http://http://www.fecolsog.org/userfiles/file/revista/Revista_Vol60No2_Abril_Junio_2009/v60n2a07.pdf

Chacón, S. (2008). La indigencia y ¿el Estado? Vuelta en U. San José, Costa Rica Recuperado de http://www.vueltaenu.co.cr/index.php?option=com content\&task=view\&id=2000

(CICAES) Comisión Intersectorial Contra el Abuso Sexual y la Explotación Sexual Comercial de Niños, Niñas y adolescentes. (2006). Plan de acción nacional contra el abuso sexual y la explotación sexual comercial de niños, niñas y adolescentes. Recuperado de http://www.annaobserva.org/ckfinder/userfiles/files/POLITICAS\%20P\%C3\%9ABLICAS\%20VENEZUELA.pdf

Curtis, G., Schulder, J. (2003). El embarazo después de los 35. Barcelona: Editorial Paidós Ibérica.

Delgado, R., López, G. (2004). Obstetricia y Ginecología. Barcelona: Editorial Ariel.

Gómez, M. (s.f.). Guía y control del embarazo en atención primaria. Recuperado de http://www.laalamedilla.org/GUIAS/Embarazo.pdf

Grandi, C., Luchtemberg, G., Sola, A. (2007). Evaluación nutricional durante el embarazo. Nuevo estándar. Medicina 67, 677-684. Recuperado de http://www.scielo.org.ar/pdf/medba/v67n6/v67n6a01.pdf

Larraín, F. (2008) Cuatro millones de pobres en chile: actualizando la línea de pobreza. Revista Estudios Públicos. $109,5-267$.

Loaiza, V. (2007). Indigentes y ladrones, “dueños” de céntricas calles capitalinas. La Nación. San José, Costa Rica.

Ministerio de Salud de Costa Rica, Organización Mundial de la Salud y Oficina Regional de la Organización Panamericana de la Salud. (2004). Las desigualdades de salud en Costa Rica: una aproximación geográfico - población. Recuperado de http://www.bvs.sa.cr/php/situacion/desigualdades.pdf

Rojas, C. (2001). Rostros de la indigencia: un estudio etnográfico en la zona noroeste del casco metropolitano de San José. Tesis de Licenciatura. Universidad de Costa Rica, San José.

Rojas, C. (2006). Indigencia en San José: expresión de la exclusión social y el desarraigo. Recuperado de http://reflexiones.fcs.ucr.ac.cr/documentos/85_1_2/indigenciaSanJose.pdf 
Rojas, L. (2008). Elementos conceptuales y metodológicos de la Investigación cualitativa: módulo de autoinstrucción. San José: Editorial Universidad de Costa Rica.

Shaw, K. (2011). Regiones Suplemento de Arqueología: El fin del 'niño de la calle' y los nuevos desafíos para la infancia excluida". Revista Regiones Suplemento de Arqueología, 8 (46), 23-27. Recuperado de

http://www.suplementoregiones.com/pdf/Regiones46.pdf\#page=23

Uauy, R.,Atalah, E., Barrera, C., Behnke, E. (2002). Alimentación y nutrición durante el embarazo. Recuperado de http://www.redsalud.gov.cl/archivos/alimentosynutricion/estrategiaintervencion/AlimentacinEmbarazo.pdf

Umbría, M. (2011). Depresión, alcohol y drogas llevan a la indigencia. Recuperado de http://www.laprensademonagas.info/Articulo.aspx?s=14\&aid=53365

Vargas, O. (2008). San José alberga a 2000 indigentes adictos a drogas. La Nación. San José, Costa Rica. 